\title{
Microstructure of (Hf-Ta-Zr-Nb)C High-Entropy Carbide at Micro and Nano/Atomic Level
}

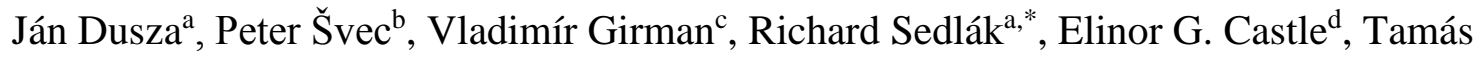
Csanádia ${ }^{a}$ Alexandra Kovalčíkováa ${ }^{a}$ Michael J. Reece ${ }^{d}$

${ }^{a}$ Institute of Materials Research, Slovak Academy of Sciences, Division of Ceramic and NonMetallic Systems, Watsonova 47, 04001 Košice, Slovak Republic

${ }^{b}$ Institute of Physics, Slovak Academy of Sciences, Dúbravská cesta 9, 84511 Bratislava, Slovak Republic

${ }^{c}$ Pavol Jozef Šafárik University in Košice, Faculty of Science, Institute of Physics, Department of Condensed Matter Physics, Park Angelinum 9, 04001 Košice, Slovak Republic

${ }^{d}$ School of Engineering \& Materials Science and Nanoforce Technology Ltd., Queen Mary University of London, Mile End Road, London E1 4NS, United Kingdom

*Corresponding author: Richard Sedlák, Institute of Materials Research, Slovak Academy of Sciences, Division of Ceramic and Non-Metallic Systems, Watsonova 47, 04001 Košice, Slovak Republic, Phone:00421 55792 2400,e-mail: rsedlak@saske.sk

\begin{abstract}
A High Entropy (Hf-Ta-Zr-Nb)C Ultra-High Temperature Ceramic (UHTC) was fabricated by ball milling and Spark Plasma Sintering (SPS) with a density of 99\%. The microstructure characteristics were investigated using X-ray diffraction (XRD), scanning electron microscopy (SEM) in combination with electron back scattered diffraction (EBSD) and transmission electron microscopy (TEM). Atomic structure and local chemical disorder was determined by means of scanning transmission electron microscopy (STEM) in conjunction with energy dispersive X-ray spectroscopy (EDS). According to the results, high purity, dense and homogeneous high entropy carbide with Fm-3m crystal structure was successfully produced. The grain size ranged from approximately $5 \mu \mathrm{m}$ to $25 \mu \mathrm{m}$ with average grain size of $12 \mu \mathrm{m}$. Chemical analyses proved that all grains had the same chemical composition at the micro as well as on the nano/atomic level without any detectable segregation. The approximately $1.5 \mathrm{~nm}$ thin amorphous grain boundary phase contained impurities that came from the starting powders and the ball milling process.
\end{abstract}


Keywords: High-Entropy Carbides, Microstructure, Grain Boundary, Chemical Composition, TEM/HRTEM

\section{- Introduction}

Since their discovery in 2004, significant research efforts have been focused on the fabrication and characterisation of metallic High Entropy Alloys (HEAs) with superior physical and mechanical properties, including: high strength/hardness, outstanding wear resistance, exceptional high-temperature strength, good structural stability and good corrosion and oxidation resistance [1-6]. HEAs typically consist of five or more elements that are mixed in equimolar concentrations to produce a maximum molar configurational entropy of $\Delta \mathrm{Smix}=\mathrm{R} \ln \mathrm{N}$, where $\mathrm{N}$ is the number of equimolar components and $\mathrm{R}$ is the gas constant [7]. This entropic term has the effect of stabilising single phase multicomponent solid solutions.

The large body of research undertaken in the field of HEAs has significantly improved the understanding of these new materials and intensive research is underway to develop HEAs as structural materials for e.g. high-temperature applications, hydrogen storage materials, radiation resistant materials, diffusion barriers for electronics, precision resistors, electromagnetic shielding materials, soft magnetic materials, thermoelectric materials, functional coatings, anti-bacterial materials etc. [7]. HEAs are of interest for these applications for a number of reasons. The most obvious is that the compositional space available for the exploration and discovery of new materials is vastly increased due to the greater number of components. Most compositions will not form pure, fully chemically disordered single phases (the equiatomic compositions will have the highest entropy of mixing and hence will be the most "entropy stabilized") however the formation of second phases, precipitates, local chemical ordering etc. can offer additional routes to property enhancement. In the materials where single phases do form from multiple elements, the chemical and structural disorder introduced into these materials can significantly influence dislocation behaviour, as well as mass, heat and charge transport, leading to materials properties that are enhanced beyond that which would be expected from a rule of mixtures approximation [8].

Due to their more recent discovery, only a handful of bulk high entropy ceramics (HECs) have been successfully fabricated in bulk form [9-11], and hence there is still much to investigate about these materials and their properties.

Rost et al. were the first to report a bulk entropy-stabilized rock salt (Fm-3m crystal structure) oxide and its derivative materials doped with $\mathrm{Li}$ and $\mathrm{Ga}$ cations, which showed promising ion-conducting and dielectric properties [9]. Gild et al. [10] then successfully 
synthesized six single-phase, high-entropy, metal diborides using mechanical alloying and SPS, with densities of $>92 \%$ and largely uniform compositions at the nanoscale and microscale. They observed the formation of 2D high-entropy metal layers, separated by rigid 2D boron nets, without any detectable segregation between the layers measured along the c-axis. Initial property assessments showed that both the hardness and the oxidation resistance of these highentropy metal diborides were generally higher/better than the average performances of the five individual metal diborides made using the same processing route.

More recently Jiang et al. [11] reported the first successful synthesis of high-entropy perovskite oxides (i.e. single solid-solution phases of multi-cation perovskite oxides with high configurational entropies of $\geq 1.5 \mathrm{R}$ per mole). They found that the Goldschmidt's tolerance factor, instead of cation-size difference, influences the formation and temperature-stability of the single phase cubic perovskite solid solutions. The discovery of this new class of multi-cation (high-entropy) perovskite solid solutions may allow the simultaneous tailoring of multiple physical properties.

The carbides of refractory metals $\mathrm{TiC}, \mathrm{ZrC}, \mathrm{HfC}, \mathrm{NbC}$ and $\mathrm{TaC}$ have attracted considerable attention for their excellent physical, chemical and mechanical properties. Of these, $\mathrm{TaC}$ and $\mathrm{HfC}$ are the most refractory, with melting temperatures approaching $4000{ }^{\circ} \mathrm{C}$. The refractory carbides also exhibit high hardness, strength, and wear resistance thanks to their mixed covalent-metallic-ionic chemical bonding [12-19]. Various compositions of TaC-HfC compounds have been prepared by different processing routes, such as hot pressing, hot isostatic pressing or spark plasma sintering, with the aim to increase their melting point, hardness and reliability [12-15].

Omar Cedillos-Barraza et al. [16] reported solid solution formation and densification by a solid-state mechanism for $4 \mathrm{TaC}-1 \mathrm{HfC}, 1 \mathrm{TaC}-1 \mathrm{HfC}$ and $1 \mathrm{TaC}-4 \mathrm{HfC}$ powders during SPS sintering at temperatures of at least $2350{ }^{\circ} \mathrm{C}$. Phase analysis and lattice parameter measurements using X-ray diffraction (XRD) showed mutual diffusion of Hf and Ta with increasing sintering temperature. Elemental analysis using EDS in both SEM and STEM, confirmed the homogeneous distribution of $\mathrm{Ta}$, Hf and $\mathrm{C}$.

Smith et al. [19] prepared a series of HfxTa1-xC atomic compositions (where $\mathrm{x}=0.0$, $0.13,0.25,0.50,0.75,0.83$, and 1.0) and, using computational and experimental methods, studied their phase, hardness and dislocation emission behaviour in order to determine how the composition of the mixed metal species within the ternary metal carbides alters the mechanical properties and the active slip systems. It was found that even small amounts of Ta added into 
the HfC rich composition changes the preferred slip system from $\langle 1-10\rangle\{110\}$, as reported for pure HfC, to $\langle 1-10\rangle\{111\}$ due to the introduction of a stacking faults on the $\{111\}$ planes.

We have recently reported the fabrication of (Hf-Ta-Zr-Ti)C and (Hf-Ta-Zr-Nb)C high entropy Ultra-High Temperature Ceramic (UHTC) carbide compositions via ball milling and Spark Plasma Sintering (SPS) [20]. We found that the lattice parameter mismatch of the component monocarbides is a key predictive factor for determining solid solution formation, and hence the (Hf-Ta-Zr-Nb)C composition formed a single phase solid solution more readily than the (Hf-Ta-Zr-Ti)C composition. The indentation modulus of the single phase (Hf-Ta-Zr$\mathrm{Nb}) \mathrm{C}$ system was found to be slightly higher $(598 \mathrm{GPa})$ than the corresponding rule of mixtures modulus calculated from the individual component carbides while a significant enhancement in hardness was observed due to solid solution strengthening, giving a value of $36.1 \mathrm{GPa}$, equating to a $\sim 30 \%$ increase in comparison to the corresponding rule of mixtures prediction.

The aim of the present contribution is to provide a more detailed description of the microstructure of the recently developed (Hf-Ta-Zr-Nb)C HEC system at micro and nano/atomic level.

\section{Experimental material and methods}

The details of the materials processing can be found in [20]. Here we describe the optimized processing route used to produce a pure, single phase, high density (99\%) (Hf-Ta$\mathrm{Zr}-\mathrm{Nb}$ )C HEC. The selected carbide powders were weighed in equiatomic proportions, taking into account the relatively large amount of impurity $\mathrm{Hf}$ in the $\mathrm{ZrC}$ powder, and mixed by ball milling at $200 \mathrm{rpm}$ for $24 \mathrm{hr}$ ( 5 min milling, $5 \mathrm{~min}$ pause) in WC pots, using SiC milling media, stearic acid as a process control agent and a ball-to-powder ratio of 5:1. The powder mixtures were sintered by Spark Plasma Sintering (SPS) (FCT HPD 25; FCT Systeme GmbH, Rauenstein, Germany). A pressureless degassing stage was first applied, heating the powder to $1800{ }^{\circ} \mathrm{C}$ for $10 \mathrm{~min}$. Following this a two-step heating profile with a $10 \mathrm{~min}$ dwell at $1800{ }^{\circ} \mathrm{C}$ and a 7 min dwell at $2300{ }^{\circ} \mathrm{C}$ was applied under a maximum $40 \mathrm{MPa}$ uniaxial pressure.

Specimens for microstructure examination were cut, ground and polished to produce a better than $1 \mu \mathrm{m}$ final finish. The grain size distribution of the HEC grains was determined using a FEI Quanta 3D scanning electron microscope (SEM) equipped with a back scattered diffraction (EBSD) detector. The investigated HEC material has a cubic $\mathrm{B} 1 \mathrm{NaCl}$ crystal structure (space group Fm-3m, No. 225), confirmed by XRD [20], which was used as an imput structural parameter for the indexing of the EBSD patterns. The lattice parameter of ( $\mathrm{Hf}-\mathrm{Ta}-\mathrm{Zr}-\mathrm{Nb}) \mathrm{C}$ was $\mathrm{a}=0.4553 \mathrm{~nm}$, also determined from the XRD data collected from the sintered HEC sample. Compositional uniformity was investigated using scanning electron microscopy (SEM) images 
and energy dispersive X-ray (EDS) spectroscopy on a Zeiss Auriga machine applying an acceleration voltage of $15 \mathrm{kV}$ and acquisition time of $20 \mathrm{~min}$.

Thin foils for TEM and STEM observations were prepared using standard preparation methods including cutting, grinding, polishing and dimple grinding. The final step of the thinning procedure was done by either a standard ion mill operated at $4.5 \mathrm{kV}$ (PIPS 691, Gatan) for TEM observations or by cryo ion milling together with final polishing at $1.5 \mathrm{kV}$ (Low angle Ion milling and polishing system 1010, Fischione) for STEM imaging. The estimated foil thickness was $\sim 40 \mathrm{~nm}$ to $50 \mathrm{~nm}[21]$.

Atomic-resolution structural characterization was carried out using an aberration corrected FEI Titan Themis 300 microscope in STEM mode. The microscope was operated at $200 \mathrm{kV}$ acceleration voltage and the probe-forming optics were adjusted to form a $\sim 1 \AA$ electron probe with a convergence of $16 \mathrm{mrad}$ and beam current of approximately $50 \mathrm{pA}$ used for imaging and $90 \mathrm{pA}$ for EDS analysis respectively. The HAADF (High Angle Annular Dark Field) detector semi-angles were set to 98-200 mrad and ABF (Annular Bright Field) detector semi-angles were set to $~ 12-22$ mrad. EDS acquisition was carried out using a Super-X detector system (ChemiSTEM technology). EDS spectrum images were acquired by continuously rastering serially across a defined area of the specimen, recording cumulative EDS spectra at each position. EDS chemical maps were produced by integrating the background subtracted intensity of the $\mathrm{Zr} \mathrm{K} \alpha, \mathrm{Nb} \mathrm{K} \alpha$, Hf $\mathrm{L} \alpha$ and $\mathrm{Ta} \mathrm{L} \alpha$ absorption peaks, respectively. The STEMEDS maps present the net, background corrected and fitted intensities of the shown elements, dwell time $20 \mu \mathrm{s}$.

\section{Results and discussion}

The characteristic microstructure of the investigated material is illustrated in Fig. 1a, b. The observed porosity is in good agreement with the density measurement, there are only small pores present in the microstructure with diameter up to approximately $2 \mu \mathrm{m}$. The pores are located either on the grain boundaries, intergranularly, or within the individual grains, intragranularly. No clustering of the pores was observed. There is contrast in the main SEM image and individual grains can be seen due to different crystallographic orientation and its effect on image signal, Fig. 1a.

The EBSD image in Fig. 1b shows that the individual grains with different crystallographic orientations are distributed randomly and practically no neighbouring grains were found with the same crystallographic orientation. In Fig. 2 the grain - size distribution of 
the investigated HEC is illustrated. The grain size varies from approximately $5 \mu \mathrm{m}$ to $30 \mu \mathrm{m}$ with an average grain size of $12 \mu \mathrm{m}$.

Compositional maps of the investigated system are illustrated in Fig. 3. The composition of the HEC is uniform and no secondary phase was detected in the microstructure.

The structural and compositional characterization of the solid solution down to the atomic level was realized by STEM using HAADF and ABF imaging modes. It was found that, within the volume of material investigated, the high-entropy metal sublattice, which is surrounded by the carbon sublattice, displays no significant compositional segregation. The HAADF and ABF micrographs in Fig. 4 (reconstructed from stacks of 6 frames), acquired with the electron beam parallel to the [011] zone axis, show a homogeneous solid solution phase. The resulting structure model (1x1 unit cell, Fm-3m) is shown in the upper part of the micrographs. The atomic arrangement of the $\mathrm{C}$ atoms (red dots) and $\mathrm{Hf}, \mathrm{Zr}$, $\mathrm{Nb}$ or Ta atoms (green dots) is clearly visible in the HAADF and ABF micrographs. This statement could be a bit misleading due to the scanning approach (way how the micrographs were acquired). For measurement, as above mentioned, a series of rotations of the scanning window should be done and processed accordingly! The compositional homogeneity of the investigated HEC down to the atomic level was confirmed by EDS mapping of the different metallic elements. Figure 5 confirms that Hf, $\mathrm{Nb}$, Ta and $\mathrm{Zr}$ are uniformly distributed in the lattice ([011] zone axis, Fm-3m) and no longrange, significant preferential segregation or aggregation was found throughout the scanned area.

With the aim to study the grain boundary characteristics of the new High Entropy Carbide, HAADF, ABF and EDS analyses were performed, see Fig. 6. It is worth to noting that, prior the EDS analysis the sample was tilted off [011] zone axis (grain on top of the micrograph) to avoid potential channelling. STEM micrograph in Fig. 6. was acquired prior to tilting. It can be seen that, at the interface studied between individual grains, there exists an amorphous intergranular phase with a thickness of approximately $1.5 \mathrm{~nm}$. In this grain boundary phase significant $\mathrm{C}, \mathrm{Nb}, \mathrm{Hf}$, Ta and $\mathrm{Zr}$ depletion was observed and $\mathrm{Fe}, \mathrm{Co}$ and $\mathrm{Cr}$ were observed to be present in nearly equal quantities as confirmed by the EDS results. The origin of these impurities is due to impurities in the starting powders and to the processing route, most likely the milling procedure. The presence of $\mathrm{Fe}$ is due to the starting $\mathrm{NbC}$ powder, which contained 0.21 wt.\% of iron [20], and the Co and Cr were probably introduced from the WC milling jars.

The absence of $\mathrm{C}$ in this grain boundary layer suggests that the elements are present in their pure metal form, rather than as carbides. The Ellingham diagrams for the corresponding transition metal carbides [21] show that the carbides of $\mathrm{Fe}, \mathrm{Co}$ and $\mathrm{Cr}$ have much more positive 
Gibb's energies of formation (are much less thermodynamically stable) than the Hf, Ta, Zr and $\mathrm{Nb}$ carbides. It therefore seems that it would be more thermodynamically favourable for the impurity $\mathrm{Fe}$, $\mathrm{Co}$ and $\mathrm{Cr}$ species to be present as pure metals; with $\mathrm{C}$ consumed by the more favourable formation of the $\mathrm{Hf}, \mathrm{Ta}, \mathrm{Zr}$ and $\mathrm{Nb}$ carbides, whose phase diagrams indicate that the rock salt structure can accommodate large $\mathrm{C}$ excesses [22]. In this case, at the $2300{ }^{\circ} \mathrm{C}$ sintering temperature employed to make the high entropy carbides, the $\mathrm{Fe}, \mathrm{Co}$ and $\mathrm{Cr}$ would be in the liquid state and would partition to the grain boundaries, as we have observed. Grain boundary phases/films, with similar thickness as in the present study have observed during the last decades in different ceramic systems [23-25].

The microstructural characteristics of the presented high entropy carbide system can be compared with the microstructures of $\mathrm{TaC}-\mathrm{HfC}$ based solid solutions reported recently, [16,19]. Omar Cedillos-Barraza et al. [16] prepared a 4TaC-1HfC system sintered by SPS at $2450{ }^{\circ} \mathrm{C}$ with relative density of $97.7 \%$ with average grain size of $7.1 \mu \mathrm{m}$. The systems prepared by Chase J. Smith [19], using HIP technology in the temperature interval $1875-2000{ }^{\circ} \mathrm{C}$ under pressures of 29.5-30 ksi, had a high density $>97.5 \%$ and low average grain sizes between 1$12 \mu \mathrm{m}$. Elemental analysis of the 4TaC-1HfC, using EDS in both the SEM and TEM, confirmed the homogeneous distribution of Ta, Hf and C and HRTEM revealed clean grain boundaries with no obvious secondary or amorphous phase present, thus suggesting densification based on solid state sintering.

Overall (within the resolution of the techniques employed) the compositional homogeneity at the micro and nanoscale of the present high entropy carbide has been shown to be uniform, asides from the small quantity of impurities that segregated to the grain boundaries.

\section{- Conclusions}

In this study the microstructure of a new type of Ultra-High Temperature Ceramic material, a high entropy (Hf-Ta-Zr-Nb)C, was characterized at the micro and nano/atomic level. The results show that:

- It is a high purity, dense and homogeneous high entropy carbide with Fm-3m crystal structure;

- $\quad$ The grain size varies from approximately $5 \mu \mathrm{m}$ to $25 \mu \mathrm{m}$, with an average grain size of $12 \mu \mathrm{m}$;

- All of grains show the same chemical composition, with the distribution of the elements shown to be homogeneous at the micro as well as at the nano level; 
- An approximately $\sim 1.5 \mathrm{~nm}$ thin amorphous intergranular phase contains impurities (Fe, $\mathrm{Co}, \mathrm{Cr}$ ) that come from the starting powders and the ball milling process.

\section{Acknowledgements}

Support from the projects APVV-15-0469, APVV-15-0621, VEGA 2/0163/16, and VEGA 2/0082/17 is acknowledged. MJR and EGC acknowledge the support of EPSRC grant XMAT $(\mathrm{EP} / \mathrm{K} 008749 / 2)$.

\section{References}

[1] B. Cantor, I.T.H. Chang, P. Knight, A.J.B. Vincent, Microstructural development in equiatomic multicomponent alloys, Mater. Sci. Eng. A. 375-377 (2004) 213-218.

[2] J.W. Yeh, S.K. Chen, S.J. Lin, J.Y. Gan, T.S. Chin, T.T. Shun, C.H. Tsau, S.Y. Chang, Nanostructured high-entropy alloys with multiple principal elements: Novel alloy design concepts and outcomes, Adv. Eng. Mater. 6 (2004) 299-303.

[3] B.S. Murty, High-entropy alloys, Butterworth-Heinemann, 2014.

[4] M.-H. Tsai, J.-W. Yeh, High-Entropy Alloys: A Critical Review, Mater. Res. Lett. 2 (2014) 107-123.

[5] Y. Zhang, T.T. Zuo, Z. Tang, M.C. Gao, K.A. Dahmen, P.K. Liaw, Z.P. Lu, Microstructures and properties of high-entropy alloys, Prog. Mater. Sci. 61 (2014) 193.

[6] Z.P. Lu, H. Wang, M.W. Chen, I. Baker, J.W. Yeh, C.T. Liu, T.G. Nieh, An assessment on the future development of high-entropy alloys: Summary from a recent workshop, Intermetallics. 66 (2015) 67-76.

[7] D.B. Miracle, O.N. Senkov, A critical review of high entropy alloys and related concepts, Acta Mater. 122 (2017) 448-511.

[8] E.J. Pickering, N.G. Jones, High-entropy alloys: a critical assessment of their founding principles and future prospects, Int. Mater. Rev. 61 (2016) 183-202.

[9] C.M. Rost, E. Sachet, T. Borman, A. Moballegh, E.C. Dickey, D. Hou, J.L. Jones, S. Curtarolo, J.P. Maria, Entropy-stabilized oxides, Nat. Commun. 6 (2015) 1-8.

[10] J. Gild, Y. Zhang, T. Harrington, S. Jiang, T. Hu, M.C. Quinn, W.M. Mellor, N. Zhou, K. Vecchio, J. Luo, High-Entropy Metal Diborides: A New Class of High-Entropy Materials and a New Type of Ultrahigh Temperature Ceramics, Sci. Rep. 6 (2016) 2 11. 
[11] S. Jiang, T. Hu, J. Gild, N. Zhou, J. Nie, M. Qin, T. Harrington, K. Vecchio, J. Luo, A new class of high-entropy perovskite oxides, Scr. Mater. 142 (2018) 116-120.

[12] D. Sciti, S. Guicciardi, M. Nygren, Densification and mechanical behavior of HfC and HfB2 fabricated by spark plasma sintering, J. Am. Ceram. Soc. 91 (2008) 1433-1440.

[13] Fischer, J.J., Hot-pressing mixed carbides of Ta, Hf, and Zr, Am. Ceram. Soc. Bull. Vol: 43 (1964) 183-185.

[14] B.C. Schulz, B. Wang, R.A. Morris, D. Butts, G.B. Thompson, Influence of hafnium carbide on vacuum plasma spray processed tantalum carbide microstructures, J. Eur. Ceram. Soc. 33 (2013) 1219-1224.

[15] S.A. Ghaffari, M.A. Faghihi-Sani, F. Golestani-Fard, H. Mandal, Spark plasma sintering of TaC-HfC UHTC via disilicides sintering aids, J. Eur. Ceram. Soc. 33 (2013) 1479-1484.

[16] O. Cedillos-Barraza, S. Grasso, N. Al Nasiri, D.D. Jayaseelan, M.J. Reece, W.E. Lee, Sintering behaviour, solid solution formation and characterisation of $\mathrm{TaC}, \mathrm{HfC}$ and TaC-HfC fabricated by spark plasma sintering, J. Eur. Ceram. Soc. 36 (2016) 15391548.

[17] O. Cedillos-Barraza, D. Manara, K. Boboridis, T. Watkins, S. Grasso, D.D. Jayaseelan, R.J.M. Konings, M.J. Reece, W.E. Lee, Investigating the highest melting temperature materials: A laser melting study of the TaC-HfC system, Sci. Rep. 6 (2016) 1-11.

[18] R.A. Andrievskii, N.S. Strel'nikova, N.I. Poltoratskii, E.D. Kharkhardin, V.S. Smirnov, Melting point in systems ZrC-HfC, TaC-ZrC, TaC-HfC, Sov. Powder Metall. Met. Ceram. 6 (1967) 65-67.

[19] C.J. Smith, X.-X. Yu, Q. Guo, C.R. Weinberger, G.B. Thompson, Phase, hardness, and deformation slip behavior in mixed Hf x Ta 1-x C, Acta Mater. 145 (2018) 142-153.

[20] E.G. Castle, T. Csanádi, S. Grasso, J. Dusza, M.J. Reece, Processing and Properties of High-Entropy Ultra-High Temperature Carbides, Submitted to Sci. Rep.

[21] S. R. Shatynski. The Thermochemistry of Transition Metal Carbides. Oxidation of Metals, Vol. 13, No. 2, 1979.

[22] A. I. Gusev and A. A. Rempel. Phase Diagrams of Metal-Carbon and Metal-Nitrogen Systems and Ordering in Strongly Nonstoichiometric Carbides and Nitrides. phys. stat. sol. (a) 163, 273 (1997).

[23] E. Okunishi, H. Sawada, Y. Kondo, Experimental study of annular bright field (ABF) imaging using aberration-corrected scanning transmission electron microscopy (STEM), Micron. 43 (2012) 538-544. 
[24] J. Jung, S. Baik, Abnormal Grain Growth of Alumina: CaO Effect, J. Am. Ceram. Soc. 86 (2003) 644-49.

[25] D.R. Clarke, On the Equilibrium Thickness of Intergranular Glass Phases in Ceramic Materials, J. Am. Ceram. Soc. 70 (1987) 15-22.

\section{List of figuress}

Fig. 1 Microstructure of (Hf-Ta-Zr-Nb) C system: a) SEM; b) EBSD.

Fig. 2 Grain size distribution of individual grains.

Fig. 3 SEM image and corresponding compositional EDX maps at micro scale.

Fig. 4 STEM micrographs a) ABF, b) HAADF along the [011] zone axis (Fm-3m). The magnified inset indicates atomic positions of $\mathrm{Nb}, \mathrm{Zr}, \mathrm{Hf}$, or $\mathrm{Ta}$ (green) and $\mathrm{C}$ (red).

Fig. 5 HAADF STEM micrograph along the [011] zone axis (Fm-3m) and indicated atomically resolved EDS maps showing mostly random distribution of constituent elements.

Fig. 6 HAADF STEM micrograph along the [011] zone axis (Fm-3m) and corresponding EDS maps (outlined in red) showing constituent elements of the grains and the thin intergranular phase (top double row). HAADF, ABF micrographs revealing fine detail across the grain boundary (outlined white), Fm-3m structure overlay. 

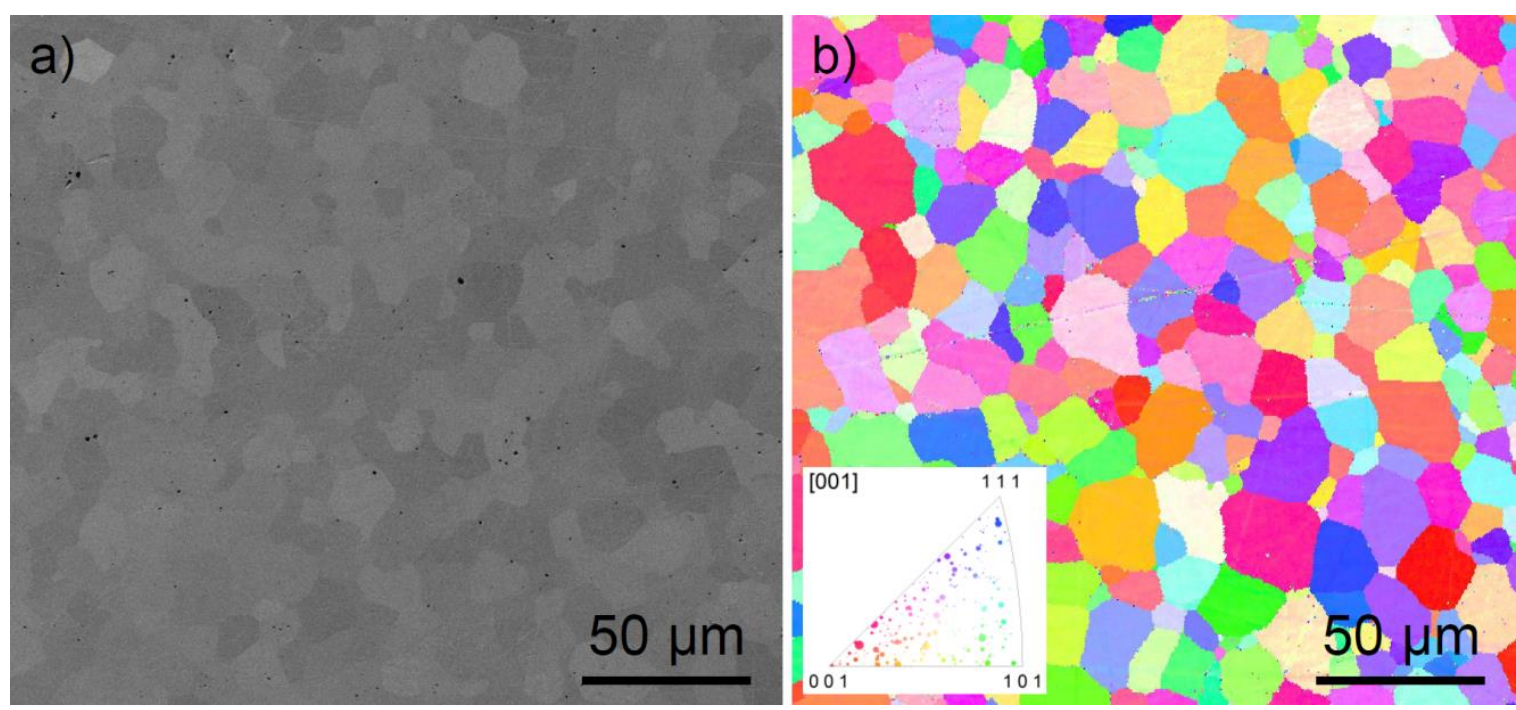

Fig. 1 Microstructure of (Hf-Ta-Zr-Nb) C system: a) SEM; b) EBSD.

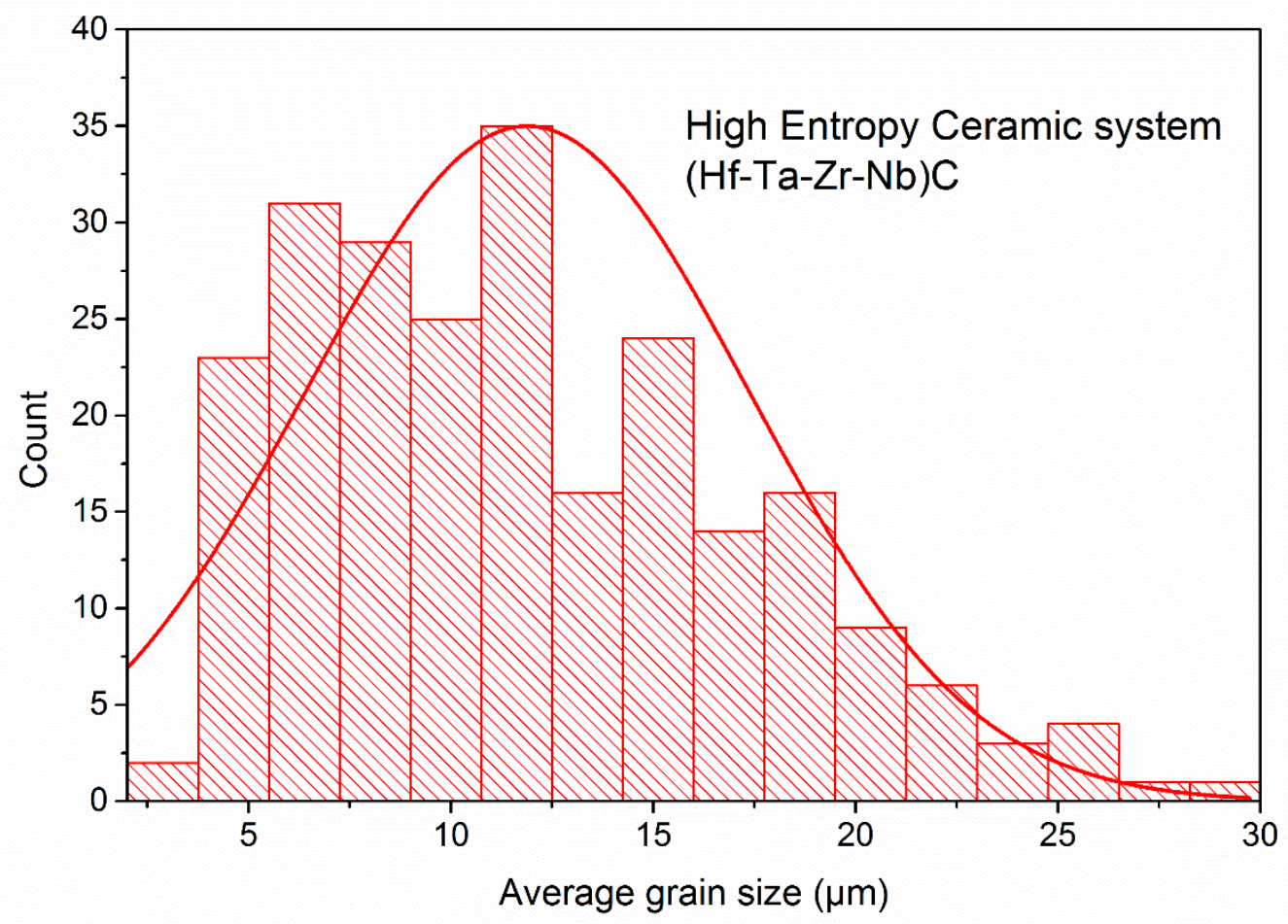

Fig. 2 Grain size distribution of individual grains. 


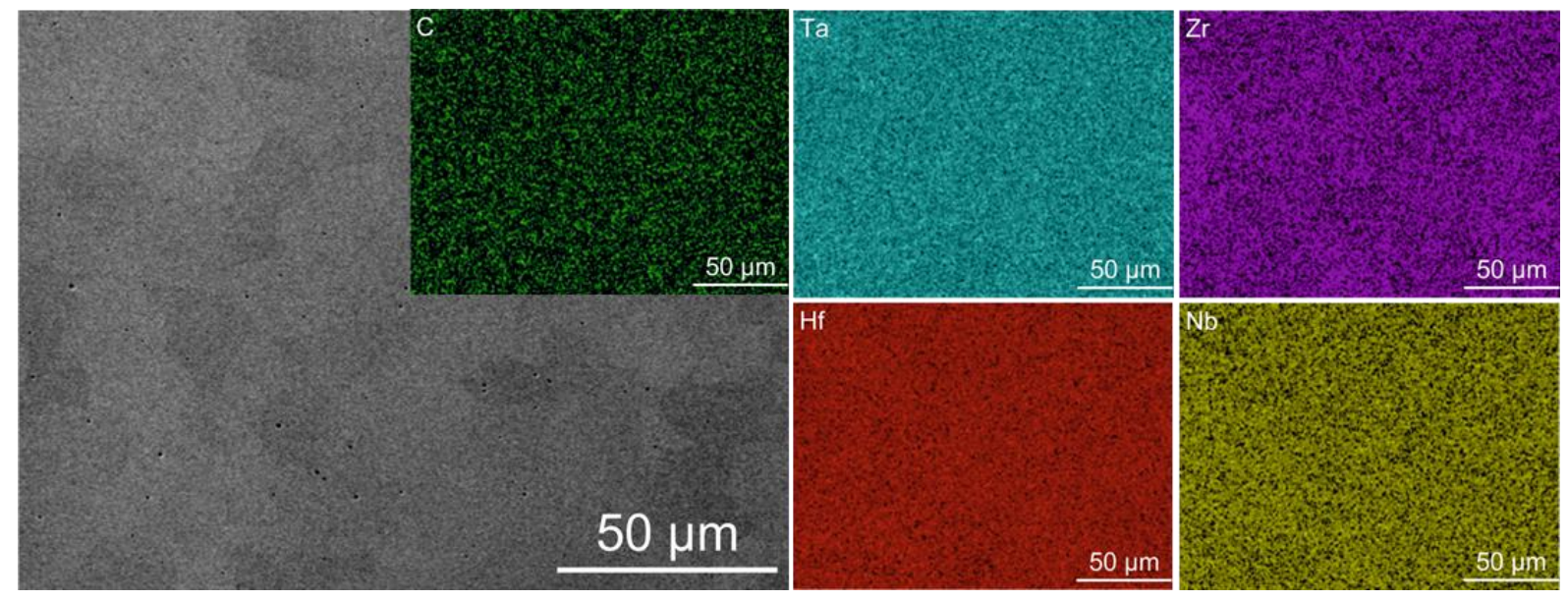

Fig. 3 SEM image and corresponding compositional EDX maps at micro scale.

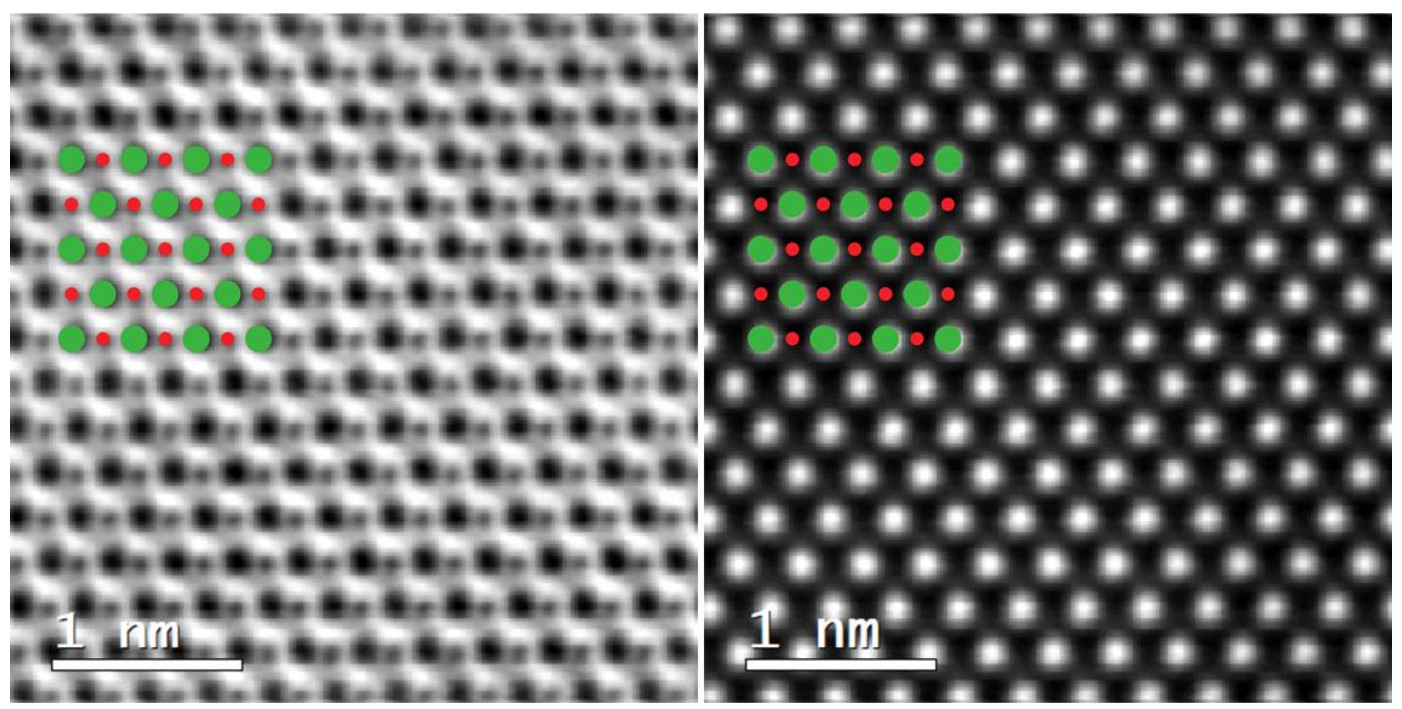

Fig. 4 STEM micrographs a) ABF, b) HAADF along the [011] zone axis (Fm-3m). The magnified inset indicates atomic positions of $\mathrm{Nb}, \mathrm{Zr}, \mathrm{Hf}$, or Ta (green) and C (red). 

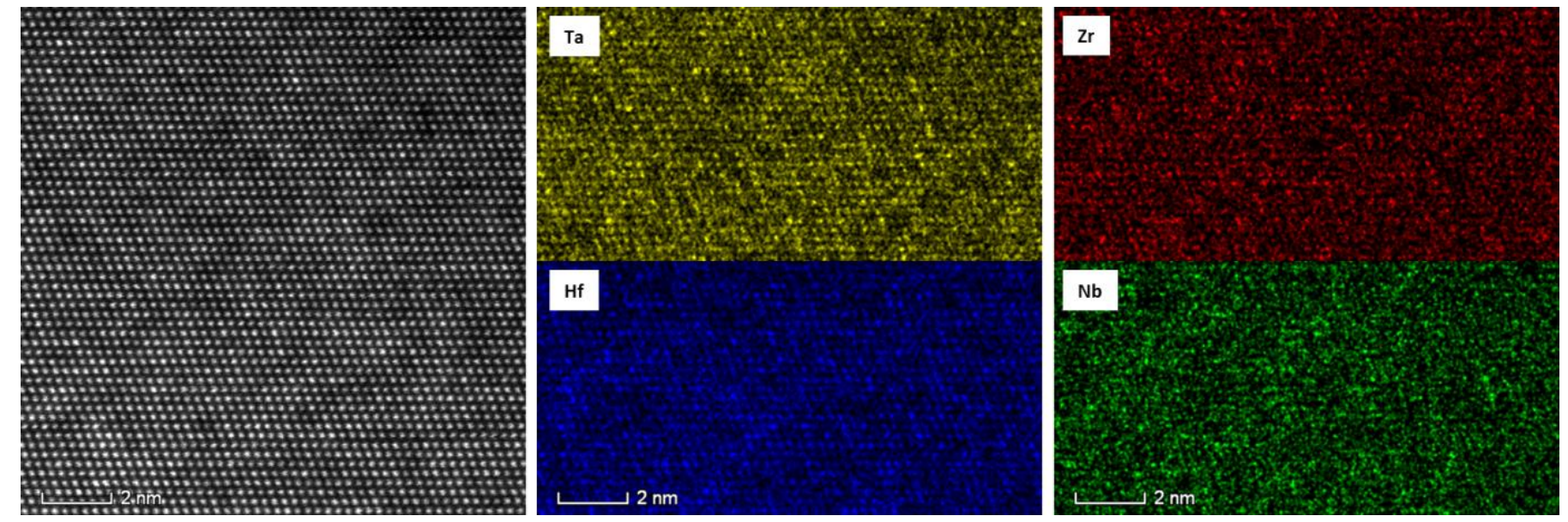

Fig. 5 HAADF STEM micrograph along the [011] zone axis (Fm-3m) and indicated atomically resolved EDS maps showing mostly random distribution of constituent elements.
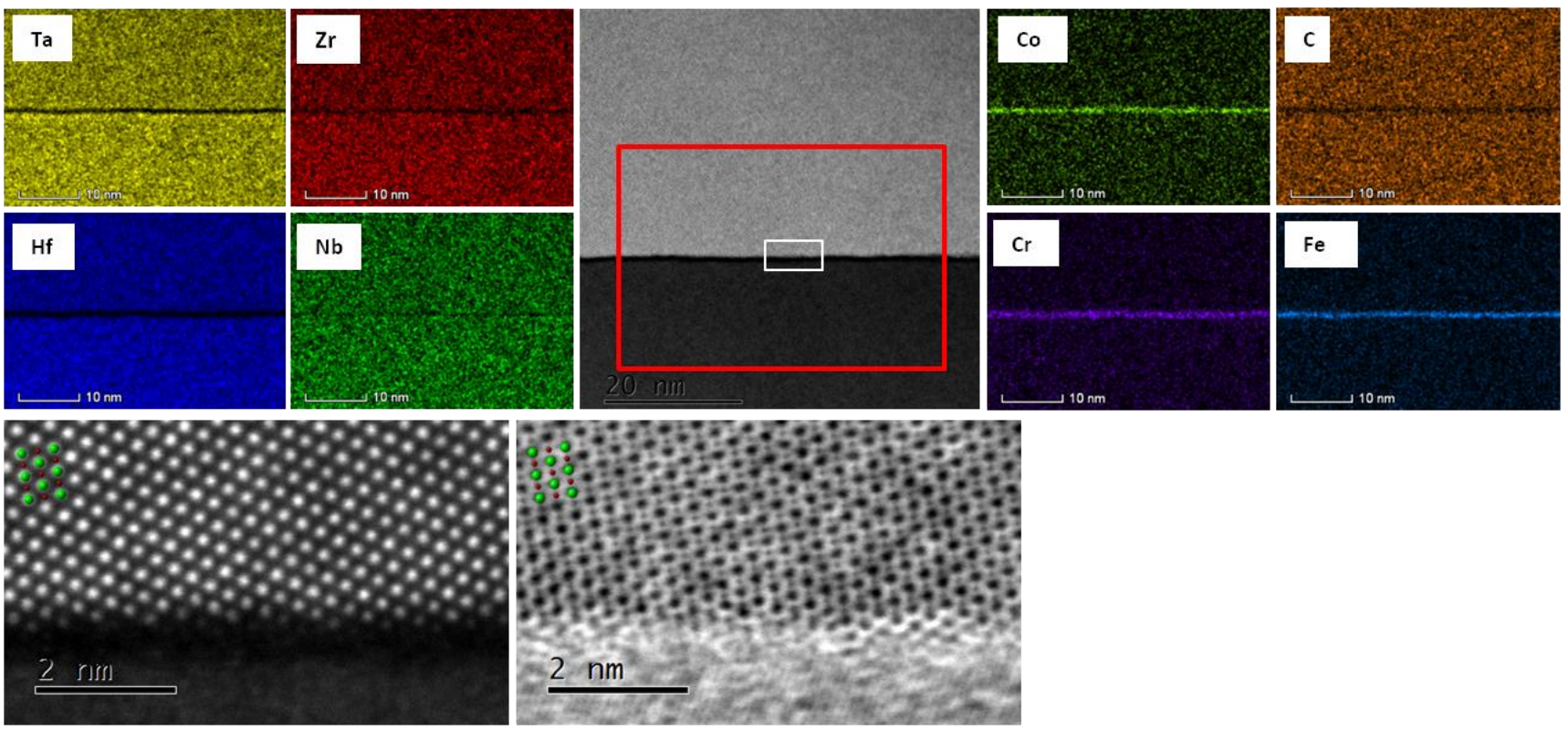

Fig. 6 HAADF STEM micrograph along the [011] zone axis (Fm-3m) and corresponding EDS maps (outlined in red) showing constituent elements of the grains and the thin intergranular phase (top double row). $H A A D F, A B F$ micrographs revealing fine detail across the grain boundary (outlined white), Fm-3m structure overlay. 